\title{
Parotid lymph node metastasis in a patient with tongue cancer
}

\author{
Kenji Yamagata*, Naomi Ishibashi Kanno, Hiroyuki Ito, Shogo Hasegawa, Toru Yanagawa, \\ Kojiro Onizawa, Hiroki Bukawa
}

Department of Oral and Maxillofacial Surgery, Faculty of Medicine, University of Tsukuba, Tsukuba, Japan;

*Corresponding Author: y-kenji@md.tsukuba.ac.jp

Received 25 November 2013; revised 22 December 2013; accepted 8 January 2014

Copyright (c) 2014 Kenji Yamagata et al. This is an open access article distributed under the Creative Commons Attribution License, which permits unrestricted use, distribution, and reproduction in any medium, provided the original work is properly cited. In accordance of the Creative Commons Attribution License all Copyrights (C) 2014 are reserved for SCIRP and the owner of the intellectual property Kenji Yamagata et al. All Copyright (C) 2014 are guarded by law and by SCIRP as a guardian.

\section{ABSTRACT}

Parotid lymph node (LN) metastasis occurs most commonly from cutaneous squamous cell carcinoma (CSCC) of the head and neck, but rarely from oral squamous cell carcinoma (OSCC). Here, we present a rare case of metastasis to the parotid LN in a patient with tongue cancer. Although usual extent of modified radical neck dissection that includes resection of the tail of the parotid gland is below the line of the mandibular angle and the mastoid process was performed, the parotid LN metastasis occurred. The patient was treated with partial parotidectomy and chemoradiotherapy. One year and two months later, there was no evidence of local recurrence, although multiple lung metastases were observed. We also review the literature on parotid LN metastasis from OSCC.

\section{KEYWORDS}

\section{Oral Squamous Cell Carcinoma (OSCC); Parotid Lymph Node Metastases; Tongue Cancer}

\section{INTRODUCTION}

Parotid lymph node (LN) metastasis occurs most frequently from cutaneous squamous cell carcinoma (CSCC) of the head and neck but may also arise from non-cutaneous regions of the head and neck or from distant sites [1]. In CSCC of the head and neck, metastatic involvement of the parotid gland is associated with a poor prognosis [2]. The parotid LN is an uncommon site of metastasis for oral squamous cell carcinoma (OSCC). To our knowledge, only 34 OSCC cases of LN metastasis from
OSCC have been previously reported (in 5 papers), and only 10 of these cases originated from tongue SCC [3-7]. We present here a rare case of metastasis to the parotid LN in a patient with tongue SCC and a review of the literature.

\section{CASE REPORT}

A 59-year-old Japanese man was referred to the Department of Oral and Maxillofacial Surgery, University of Tsukuba Hospital, in December 2008. He complained of an ulcer in his right tongue that had been there for one month. The medical history was hypertension, premature ventricular contraction, and herniated disc. The clinical diagnosis was tongue cancer (T2N2bM0, StageIV). In January 2009, a partial glossectomy with modified radical neck dissection (MRND) was performed under general anesthesia. The tail of the parotid gland below the line of the mandibular angle and the mastoid process was resected; in this procedure, the mandibular branch of the facial nerve was identified and preserved. The histopathological diagnosis was moderate SCC with free surgical margins. Two metastatic LNs were observed among the superior internal jugular nodes.

Nine months later, magnetic resonance imaging (MRI) depicted a growing right parotid LN (Figure 1). In addition, 18F-fluorodeoxy-glucose positron-emission tomography combined with computed tomography (18F-FDG PET/CT) revealed FDG uptake in the tail of the right parotid gland. There was no FDG uptake outside the parotid gland (Figure 2). The ultrasonography (US) Bmode image showed three consecutive LNs measuring $7.5 \times 3.5 \mathrm{~mm}, 14.3 \times 7.8 \mathrm{~mm}$, and $5.4 \times 4.6 \mathrm{~mm}$ in the parotid gland. The clinical diagnosis was metastases to the parotid LNs from tongue cancer.

In November 2009, partial parotidectomy of the 3 af- 


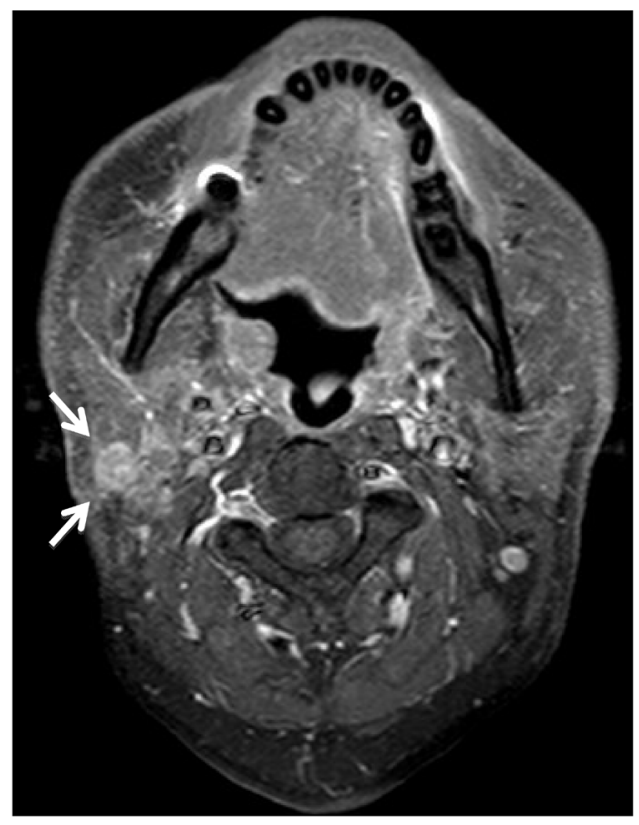

Figure 1. MRI (T1 weighted) depicts the enhanced diameter of an 11-mm LN in the right parotid gland (arrows).

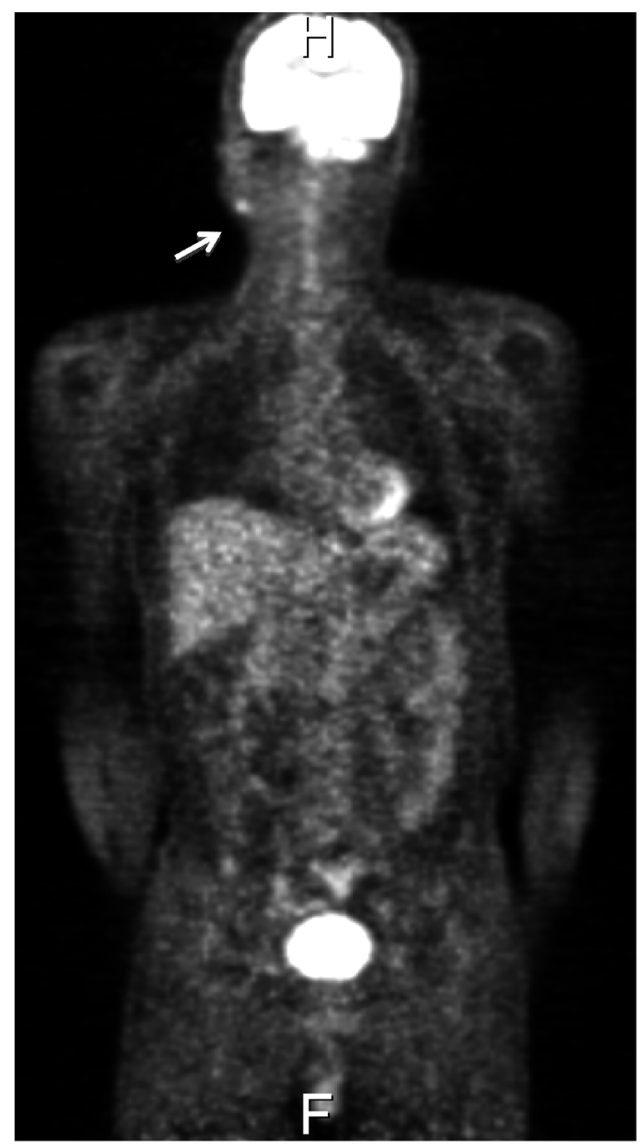

Figure 2. 18F-FDG PET/CT reveals FDG uptake in the tail of the right parotid gland (arrow). No other abnormal uptake is indicated. fected LNs detected by US was performed using intraoperative US examination (Figures 3(a) and (b)). The pathological diagnosis was that 3 of 9 excised parotid LNs were metastases of SCC with cancer pearls (Figures 4(a) and (b)). Postoperative adjuvant chemo radio therapy consisted of a total dose of 50 Gy external radiation and chemotherapy with $600 \mathrm{mg} /$ day $5 F U$ (day 1 to 6) and $135 \mathrm{mg}$ CDDP (day 7). There was no evidence of local recurrence 1year and 2 months after the partial parotidectomy, although the chest CT depicted multiple lung metastases.

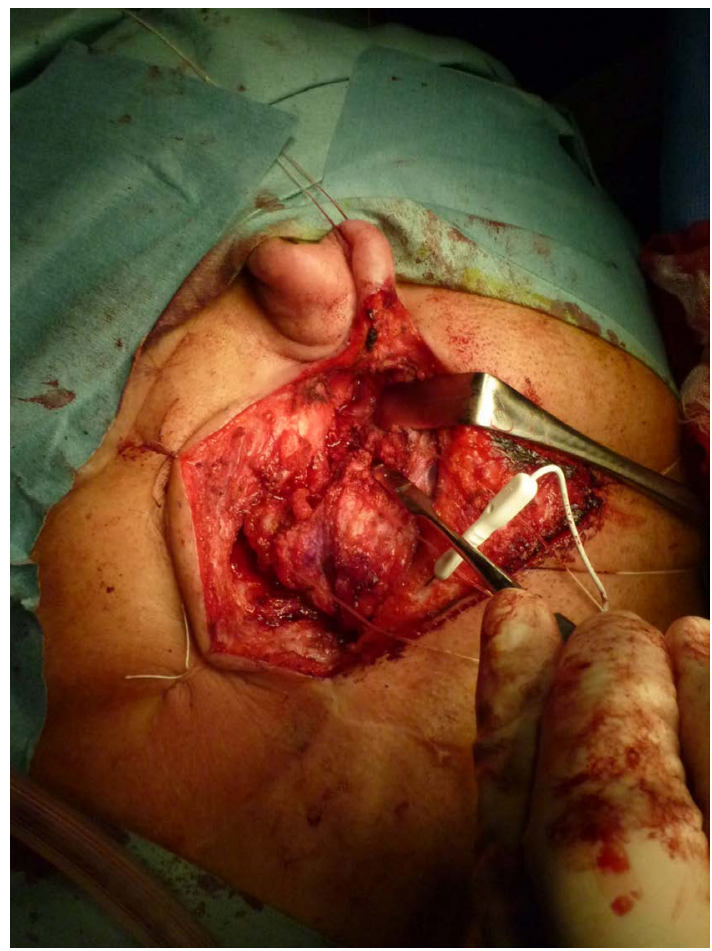

(a)

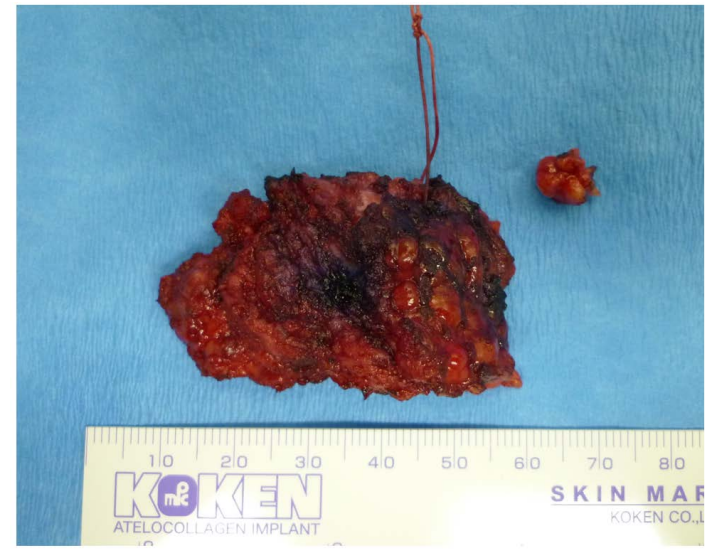

(b)

Figure 3. (a) Intra-operative photography before resection of parotid grand; (b) Clinical photograph of the resected parotid gland. Three metastatic LNs are included in this specimen. 

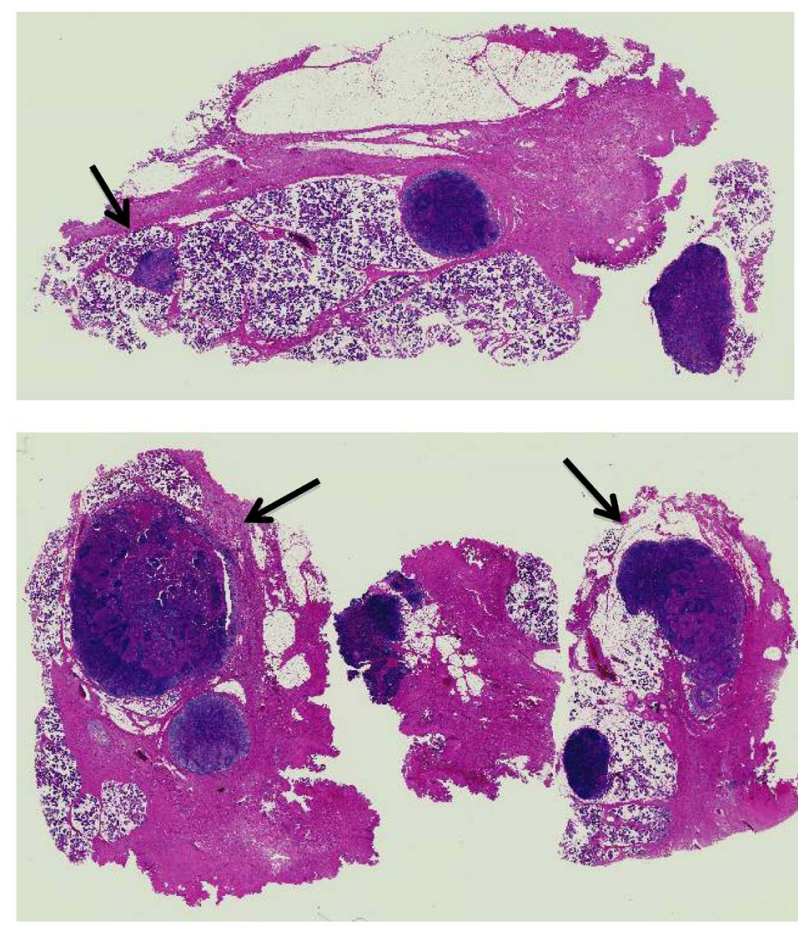

(a)

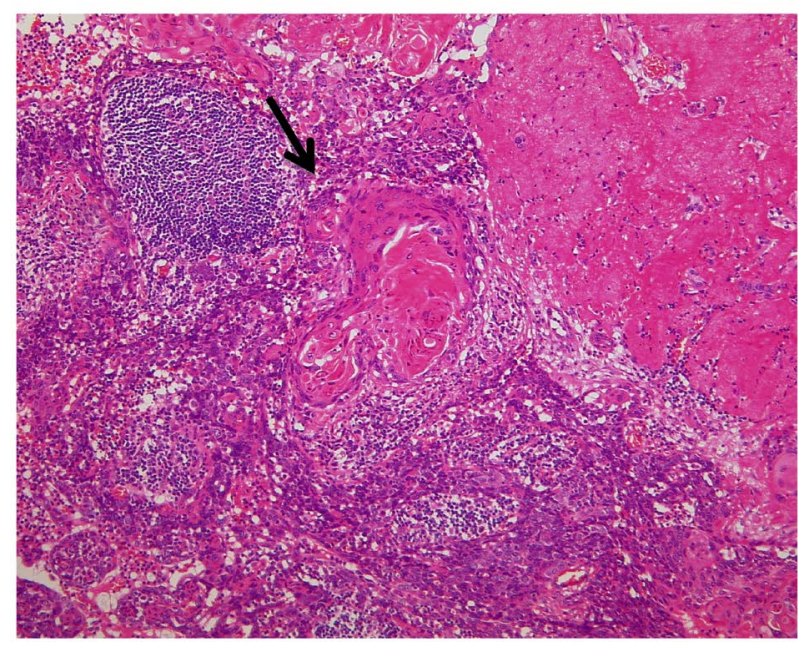

(b)

Figure 4. (a) Histological findings of surgical specimens stained with hematoxylin and eosin with megaloscope. Three LN metastases are observed (arrows); (b) Histological examination shows metastatic LNs of SCC with cancer pearls (arrow) $(\times 100)$.

\section{DISCUSSION}

There are about 15 to 20 LNs in the region of the parotid gland. According to their position in relationship to the parotid gland, they are classified as extrafascial, paraglandular, and intraglandular [8]. The anatomical drainage pathway to the parotid gland-related LNs is not clearly defined in the literature. Most tumors that metastasize to the parotid LNs are CSCCs of the head and neck, and the primary sites are the scalp, eyelid, and ear [5]. The parotid LN is an uncommon site of metastasis in OSCC. To our knowledge, six papers including the present one have reported 35 cases of parotid LN metastasis from OSCC, from 1963 to 2013 [3-7]; these include 11 cases of tongue SCC (Table 1). Although Harada et al reported the 5-year incidence of parotid node metastasis for OSCC patients to be $2.5 \%$ [5], it was $0.51 \%(1 / 197)$ in our department. The reported primary site of the parotid LN metastasis was the tongue in 11 patients, the gingiva in 10 , the buccal mucosa in 9 , and other in 5 . The clinical stage was I for 3 patients, II for 3, III for 6, and IV for 17. Thus, the cases of parotid LN metastasis most often involved cancer at an advanced stage (Table 1).

Table 1. Summary of the reported clinical characteristics of parotid lymph node metastasis from OSCC including our case $(\mathrm{n}=35)$.

\begin{tabular}{|c|c|}
\hline Characteristics & No. of patients \\
\hline \multicolumn{2}{|l|}{ Primary site } \\
\hline Tongue & 11 \\
\hline Gingiva & 10 \\
\hline Buccal mucosa & 9 \\
\hline Others & 5 \\
\hline \multicolumn{2}{|c|}{ Stage (Primary treatment) } \\
\hline I & 3 \\
\hline II & 3 \\
\hline III & 6 \\
\hline IV & 17 \\
\hline ND & 6 \\
\hline \multicolumn{2}{|c|}{ Metastatic parotid site $(\mathrm{n}=18)$} \\
\hline Intraglandular & 12 (19 LNs) \\
\hline Extraglandular & 10 (18 LNs) \\
\hline \multicolumn{2}{|l|}{ Outcome } \\
\hline Alive & 5 \\
\hline Died & 24 \\
\hline ND & 6 \\
\hline \multicolumn{2}{|l|}{ Treatment $(\mathrm{n}=9)$} \\
\hline S & 5 \\
\hline $\mathrm{S}+\mathrm{R}$ & 1 \\
\hline $\mathrm{S}+\mathrm{C}+\mathrm{R}$ & 2 \\
\hline $\mathrm{C}$ & 1 \\
\hline
\end{tabular}

Abbreviations: ND, not described; LN, Lymph node; S, Surgery; R, Radiotherapy; C, Chemotherapy. 
Parotid LN metastasis is rare in OSCC patients, especially at the initial presentation. Due to this extremely low incidence, and because of the risk of facial nerve injury, dissections of unusual site metastases of the neck have been rarely performed during the initial treatment of OSCC [7]. On the other hand, it was reported that metastasis to the parotid LNs should be considered in patients with OSCC, and that because small numbers of LNs or small tumor extensions infiltrating the surrounding healthy tissue are often not palpable, resection of the parotid gland tail is warranted during the initial neck dissection [5]. Although our patient underwent MRND surgery that included resection of the tail of the parotid gland as a primary treatment, parotid LN metastases as an unusual site recurrence was observed 9 months later. Our patient had N2b LN metastasis at the time of MRND, with the secondary metastases occurring in the parotid LNs. Olsen et al.'s study of 12 cases of oral and or opharynx SCC with metastasis to the parotid showed that metastasis to the intraparotid LNs could occur, that the inferior parotid nodes were most commonly involved, and that substantial cervical metastases were often observed [6]. In that report, parotid LN metastasis was located in the inferior parotid node in 7 cases, multiple superficial nodes in 3 cases, and in both deep and superficial nodes in 2 cases [6]. The regions of parotid LN metastases have been reported for 18 OSCC patients, as 18 extraglandular nodes and 19 intraglandular nodes (Table 1) $[3,5,6]$. Our patient had an intraglandular node metastasis.

Normal pathways of lymphatic spread may be interrupted easily by even minor surgical procedures, radiotherapy, or the presence of massive metastasis in the neck $[4,9]$. The presentation of parotid LN metastasis suggests that direct lymphatic connections exist between the parotid gland and the oral cavity or the oropharynx. Alternatively, parotid LN metastases may result from retrograde or aberrant lymphatic drainage due to extensive neck disease. The involvement of three or more positive cervical nodes is reported to be a risk factor for parotid LN metastases [5]. In the present case, the patient had two LN metastases at the time of MRND. The patient presented with delayed parotid LN metastases at the cranialis of the resected parotid gland, even though MRND with resection of the inferior parotid preserving the marginal mandibular branch had been performed. Liao et al reported that, in OSCC patients, parotid LN metastasis as an unusual neck recurrence after neck dissection occurred at 21.5 months on average (1 month - 105 months) [7]. In our patient, the parotid LN metastasis occurred 8 months after MRND.

Metastasis of the parotid LN is associated with a poor prognosis in CSCC of the head and neck [2]. While the reports are rare, metastatic involvement of the parotid gland from OSCC also appears to be associated with a poor prognosis. Liao et al. reported the independent prognostic factors for 5-year disease specific survival (DSS) of OSCC to be poor differentiation, pN2, extracapsular spread, tumor depth more than $10 \mathrm{~mm}$, relapse time less than 10 months, local recurrence, distant metastases, and neck recurrence at unusual sites [7]. As unusual sites, 13 parotid areas were included [7]. Many researchers have advocated that the use of combination therapy for CSCC, that is, surgery followed by irradiation, results in better survival than treatment with either modality alone [2]. Of the 35 reported OSCC cases, 24 patients (68.6\%) had died, 5 (14.3\%) were alive, and 6 were not described. In the 11 tongue cases including ours, 7 patients had died (Table 1 ). These results indicate a poor prognosis for OSCC cases with parotid LN metastasis, similar to CSCC. The reported treatments for parotid LN metastases were surgery alone in 5 cases, surgery with chemoradiotherapy in 2, surgery with radiotherapy in 1 , and chemotherapy alone in 1 (Table 1). There is no consensus about the appropriate treatment for the parotid LN metastasis in OSCC because of the small number of reported cases. In the present case, although the resected parotid area including LN metastasis was uneventful because of the partial resection and following chemoradiotherapy, lung metastases occurred just over 1year after surgery, presenting a poor prognosis.

\section{CONCLUSION}

We presented here a rare case of metastasis to the parotid LN in a patient with tongue SCC and a review of the literature. Metastasis of the parotid $\mathrm{LN}$ is associated with a poor prognosis in SCC of the head and neck. There is no consensus on the appropriate treatment for the parotid LN metastasis in OSCC because of the small number of reported cases. The additional study of the large sample cases would be desired in the future.

\section{REFERENCES}

[1] Bumpous, J. (2009) Metastatic cutaneous squamous cell carcinoma to the parotid and cervical lymph nodes: treatment and outcomes. Current Opinion in Otolaryngology \& Head and Neck Surgery, 17, 122-125.

http://dx.doi.org/10.1097/MOO.0b013e32832924e0

[2] Audet, N., Palme, C.E., Gullane, P.J., Gilbert, R.W., Brown, D.H., Irish, J. and Neligan, P. (2004) Cutaneous metastatic squamous cell carcinoma to the parotid gland: Analysis and outcome. Head \& Neck, 26, 727-732. http://dx.doi.org/10.1002/hed.20048

[3] Conley, J. and Arena, S. (1963) Parotid gland as a focus of metastasis. Archives of Surgery, 87, 757-764. http://dx.doi.org/10.1001/archsurg.1963.01310170043009

[4] Ord, R.A., Ward-Booth, R.P. and Avery, B.S. (1989) Pa- 
rotid lymph node metastases from primary intra-oral squamous carcinomas. International Journal of Oral and Maxillofacial Surgery, 18, 104-106. http://dx.doi.org/10.1016/S0901-5027(89)80141-9

[5] Harada, H. and Omura, K. (2009) Metastasis of oral cancer to the parotid node. European Journal of Surgical Oncology, 35, 890-894. http://dx.doi.org/10.1016/j.ejso.2008.09.013

[6] Olsen, S.M., Moore, E.J., Koch, C.A., Kasperbauer, J.L. and Olsen, K.D. (2011) Oral cavity and oropharynx squamous cell carcinoma with metastasis to the parotid lymph nodes. Oral Oncology, 47, 142-144. http://dx.doi.org/10.1016/j.oraloncology.2010.11.010

[7] Liao, C.T., Lin, C.Y., Fan, K.H., Hsueh, C., Lee, L.Y., Wang, H.M., Huang, S.F., Chen, I.H., Kang, C.J., Ng,
S.H., Lin, C.H., Huang, Y.C. and Yen, T.C. (2013) Outcome analyses of unusual site neck recurrence in oral cavity cancer. Annals of Surgical Oncology, 20, 257-266. http://dx.doi.org/10.1245/s10434-012-2580-3

[8] Pisani, P., Ramponi, A. and Pia, F. (1996) The deep parotid lymph nodes: An anatomical and oncological study. The Journal of Laryngology \& Otology, 110, 148-150. http://dx.doi.org/10.1017/S0022215100133006

[9] Parsons, J.T., Million, R.R. and Cassisi, N.J. (1985) The influence of excisional or incisional biopsy of metastatic neck nodes on the management of head and neck cancer. International Journal of Radiation Oncology Biology Physics, 11, 1447-1454.

http://dx.doi.org/10.1016/0360-3016(85)90331-1 